\title{
DE HEGEMONÍAS, INSTITUCIONALIDADES Y BIG DATA: OTRA PULSIÓN DESLABORALIZADORA
}

\author{
ABOUT HEGEMONIES, INSTITUTIONALITIES AND BIG DATA: ANOTHER UNWORKING \\ DRIVE
}

\begin{abstract}
Mario L. Gambacorta
Abogado. Doctor en Ciencias Jurídicas. Docente de grado y postgrado; Investigador. UBA, UNPAZ, FLACSO, UMET, UMSA, UNLZ.

E-mail: mlgambacorta@gmail.com
\end{abstract}

Convidado

RESUMEN: El presente trabajo se orienta a constituirse en un aporte preliminar para el debate en
torno a las nuevas tecnologías y el futuro del trabajo. Particularmente, con relación a la denominada
big data. Es nuestro propósito señalar aspectos de esta temática en vista a atender nuevos escenarios
y permitir esbozar estrategias tutelares hacia lo laboral que no caigan en meras generalizaciones
antagónicas ante el discurso neoliberal prevalente. Consideramos que la big data no es solo un
cambio tecnológico, es una nueva lógica de mercado. La lógica de mercado se está reconfigurando
fuertemente y todavía, no logramos dimensionar el grado que puede alcanzar. Estas
transformaciones también se dirigen a conquistar, mantener o potenciar determinadas lógicas
institucionales. Simplificando, se está consolidando una lógica hegemónica. Por ello, es que
impulsamos reflexionar a partir del marco de un enfoque crítico tutelar de las relaciones laborales.

Palabras claves: Big data. Futuro del trabajo. Nuevas tecnologías. Hegemonía.

\begin{abstract}
This paper is aimed at becoming a preliminary contribution to the debate about new technologies and the future of work. Particularly, in relation to the so-called big data. It is our purpose to point out aspects of this theme in order to attend to new scenarios and allow to outline protective strategies towards work that do not fall into mere antagonistic generalizations before the prevailing neoliberal discourse. We considerer that big data is not just a technological change, it is a new market logic. The market logic is being strongly reconfigured and we still cannot measure the degree that it can reach. These transformations are also aimed at conquering, maintaining or promoting certain institutional logics. Simplifying, a hegemonic logic is being consolidated. For this reason, we encourage reflection from the framework of a critical protective approach to labor relations.
\end{abstract}

Keywords: Big data. Future of work. Hegemony.

SUMÁRIO: 1. Para comenzar una contextualización: neoliberalismo y hegemonía; 2. De las institucionalidades civiles, políticas ¿y sociales?; 3. Algunas referencias hegemónicas a la denominada big data; 4. La big data y la regulación; 5. Comercio electrónico, futuro del trabajo y G20; 6. Conceptos y discursos: un poco más en torno al G20; 7. Concluyendo: desafíos ante un 
nuevo modelo de acumulación; Bibliografía.

\section{PARA COMENZAR UNA CONTEXTUALIZACIÓN: NEOLIBERALISMO Y HEGEMONÍA}

Cuando se habla de tecnologías se suele prestar más atención a los aspectos económicos que a los trabajadores involucrados, sea por acción o exclusión referencial. Lo señalado precedentemente, nos lleva a recordar la hegemonía prevalente porque, aunque no se reflexione lo suficiente en torno a ello, vivimos en una hegemonía neoliberal de carácter global. Sin embargo, nadie parece tener demasiado en claro qué es el neoliberalismo. Este autor ha señalado que lo que ocurre con el neoliberalismo en el presente es como si en la URSS nadie supiera qué es el comunismo. Sintetizando, no se tiene demasiado en claro qué es el neoliberalismo, quien lo representa, y menos aún, a quiénes representa (cf. Monbiot, 2016).

Podemos sostener que $75 \%$ del mundo actual está bajo la prevalencia de los paradigmas neoliberales impulsados por Hayek y Mises (tampoco suficientemente conocidos, salvo en determinados ambientes académicos y políticos). Por contrario sensu, en virtud de experiencias históricas como el Chile de Pinochet, la Gran Bretaña de Tatcher, o los EEUU de Reagan, las políticas que afectaron Latinoamérica en la década de 1990; podríamos colegir que se trata de una lógica no muy provechosa para los pueblos (hoy llamados genéricamente, gente).

Asimismo, es indubitable luego de su referencial experiencia práctica (el thatcherismo), que el neoliberalismo tiene una hostilidad manifiesta hacia los sindicatos o, en general, hacia los trabajadores organizados para la defensa de sus intereses. Entonces, si no nos enrolamos en tal posición, es menester discutir y articular una necesaria contrahegemonía, a saber: nuevos paradigmas que pongan en crisis los del neoliberalismo.

Este último, suele presentar sus propios paradigmas como cuestiones de sentido común aunque, en realidad, representan intereses específicos. En especial, de las grandes corporaciones conocidas como empresas multi o transnacionales.

Reflexionar sobre el contenido de los paradigmas neoliberales, trae a la vez aparejada una necesidad de conocer mejor la economía para entenderla no desde la lógica o el paradigma economicista neoliberal sino con relación a su proyección en las relaciones laborales; es decir enfrentada con la lógica protectoria del derecho del trabajo.

Un paradigma contrahegemónico alternativo partiría entonces de una concepción filosófica caracterizada por la centralidad del trabajo y la concepción de la economía como no ajena pero tampoco prevalente.

El presente trabajo se orienta a constituirse en un aporte preliminar para el debate en torno a las nuevas tecnologías y el futuro del trabajo. Particularmente, con relación a la denominada big data. Es nuestro propósito señalar aspectos de esta temática en vista a atender nuevos escenarios y permitir esbozar estrategias tutelares hacia lo laboral que no caigan en meras generalizaciones antagónicas ante el discurso neoliberal prevalente.

\section{DE LAS INSTITUCIONALIDADES CIVILES, POLÍTICAS ¿Y SOCIALES?}

Las relaciones laborales viven la tensión permanente entre el capital y el trabajo.

Empezaremos refiriendo cómo suele darse la relación entre capital y trabajo, y desde allí, pasaremos a la relación trabajo y tecnología.

Debemos preguntarnos, por ejemplo, si optamos o no por el principio de prevalencia, al decir de la Doctrina Social de la Iglesia. La prevalencia final es, en tal sentido, del trabajo como actividad creadora y dignificadora de los seres humanos. Es decir, el trabajo vinculado con la dignidad de la persona, donde el capital no deja de ser un objeto, amén del fetichismo de la mercancía. 
No olvidemos que los tratados internacionales de derechos humanos parten y se sustentan en el concepto de dignidad de la persona humana; los derechos que la componen como una universalidad y son indivisibles. Por ello, y de acuerdo a cómo se integren o no los derechos, se podrá hablar de institucionalidad e institucionalidades.

Distintas institucionalidades pujan por consolidarse con relación a las problemáticas que hoy día se dan en torno a las argumentaciones represivas que están padeciendo los movimientos sindicales. Lo anterior se apoya en "una" institucionalidad que, en general $a$ ) no considera lo social; b) se limita a tutelar derechos civiles y políticos; y c) cuya respuesta al reclamo de vigencia de los derechos sociales suelen ser formas de violencia institucionalizada sin integración de derechos.

Por eso, los movimientos populares que logran acceder al gobierno del Estado suelen tener una problemática directa con la institucionalidad vigente. Esto ocurre ante ese falso sentido común que suele invocarse, pero tiende a limitarse a un enfoque de derechos políticos y civiles (sobre todo la propiedad) como necesarios, inmediatos y exigibles, pero no así hacia los sociales.

Cuando los movimientos populares, a menudo denominados populistas, llegan al gobierno del Estado, la institucionalidad, o el paradigma prevalente todavía no contempla en general los derechos sociales. Inclusive, aunque estuvieran enunciados. Estaríamos frente a lo que Garcia Linera (2012) caracteriza como institucionalidad sucia. A la que, desde un enfoque personal, nos atrevemos a caracterizar, complementariamente, como institucionalidad ilícita. Porque es legal pero es ilícita. ${ }^{1}$

Otra conceptualización de interés para formular en términos de y para el análisis y debate, es que en el marco de los paradigmas del neoliberalismo estamos viendo y viviendo un capitalismo cada vez mas libertario. Nos encontramos en un capitalismo que, necesita acceder al Estado para, desde el Estado, poder proyectar las políticas que quiere llevar adelante.

En la lógica mencionada precedentemente, se accede al Estado para debilitarlo y promover la idea de su reemplazo institucional mediante el accionar directo de las grandes corporaciones económicas. (punto seguido). Los ejércitos privados corporativos defensores de intereses de empresas transnacionales, fundamentalmente extractivas, son un claro, y tal vez, el más dramático ejemplo de esto.

Paradójicamente, la disputa por el poder es la disputa por controlar el Estado para colocarlo en dicho lugar subordinado (o nulo), valiéndose de sus potestades desregulatorias - quasi autodesregulatorias - para otorgarle potestades al sector privado, limitando facultades mediante una paradojal regulación-desregulatoria. Por eso, los gobiernos populares padecen esa institucionalidad sucia e ilícita. Se evidencia en los hechos en cuanto que, aunque llegan al gobierno no alcanzan a controlar todo el poder del Estado.

Es sabida la incidencia de ciertos actores económicos sobre el poder del Estado, amén de su negación desde un supuesto sentido común institucionalista, en el cual lo no estrictamente "formalizado" no incidiría.

Percibimos un capitalismo que reformula dinámicamente la forma de ejercer su hegemonía, de establecer sus parámetros de gobierno, de control; estructurando y redefiniendo sus paradigmas, entre otras cosas, a través de las formas y nuevos sistemas de acumulación.

\footnotetext{
${ }^{1}$ Veamos esto un poco más. Cuando se enseña derecho del trabajo en Argentina se suele decir que el despido sin causa es un hecho legal pero ilícito. Y es ilícito porque despedir a alguien sin ninguna justificación, no debería ser válido ya que es equivalente a lo que en términos de derecho civil se denominaría un delito civil; no penalmente punible pero si incorrecto y éticamente injusto y abusivo.

En términos jurídicos lo podríamos caracterizar como, al menos, un abuso del derecho. De igual modo, la institucionalidad podría revestir este carácter ilícito.
} 
Aquí hay algo que debemos considerar para el análisis y la superación de generalizaciones: la disputa de capital y trabajo, más o menos subyacente, cambia en las formas y redefine el modelo con que el capitalismo la lleva adelante.

Así las cosas, llegamos a la profundización y complementación del cambio tecnológico de la tercera revolución industrial, sobre todo mediante lo que le agrega la denominada big data.

Es sabido que la denominada cuarta revolución industrial se articula, continúa y amplía los horizontes de su predecesora. Cabe entonces intentar comprender mejor su sentido y alcances.

\section{ALGUNAS REFERENCIAS HEGEMÓNICAS A LA DENOMINADA BIG DATA}

La big data no es solo un cambio tecnológico, es una nueva lógica de mercado que no implica la desaparición de otras lógicas de mercado. Lo que estamos viendo es que, ésta se va reconfigurando fuertemente, y todavía, no logramos dimensionar el grado que puede alcanzar. Sin embargo, evidencia que apunta a conquistar, mantener o potenciar determinadas lógicas institucionales. Simplificando, la institucionalidad es lo establecido, la forma en que se organiza algo; y formal o informalmente, esta consolidando una lógica hegemónica. Cuando se menciona el hecho de las elevadas ganancias que obtienen las cuatro o cinco principales empresas de alta tecnología de la información y procesamiento de datos, no se indica que sobre esas ganancias no siempre se pagan impuestos; criterio (que a nuestro juicio evidencia un fuerte posicionamiento ideológico) que ellas impulsan para desenvolverse. En otros términos, hay allí una institucionalidad formulada, de seguir así, por la ausencia o poca incidencia regulatoria de las normas, prioritariamente, en lo tributario y en cuanto al control de la obtención y manejo de los datos.

Pero no menos importante deviene la intencionalidad de ausencia de regulaciones laborales y de la seguridad social, y especialmente, el debilitamiento de un sujeto colectivo como el sindicato.

El sistema de big data (Zubboff, 2015), se puede definir como un capitalismo de vigilancia. Dicha investigadora ha acuñado este concepto, el cual ya nos está marcando una suerte de paradigma, apoyado sobre todo, en el relevamiento, extracción y el análisis de datos, los que son extraídos, analizados y sistematizados mediante complejos algoritmos. Se trata de una lógica con un requerimiento cada vez mayor de datos vinculada con la estructuración de monopolios, aunque y paradójicamente, se nos hable de la libertad de mercado.

También es categorizable como un tecnolibertarismo (cf. Sadin, 2018: 123).

Lo entendemos como una suerte de anarquismo, caracterizable, en términos clásicos, como de derecha por su carácter y proximidad a la lógica neoliberal, pero que la profundiza en su limitación de lo estatal.

En esta instancia cabe preguntarnos ¿cuáles son y si hay alternativas a esa forma de llevar adelante esta lógica no solo digital sino sobre todo de mercado?

Recordemos que el trabajo y el capital —en sistemas de relaciones laborales como el argentino - tratan de convivir, pero no abjuran por ello del conflicto subyacente. Además, ante esta lógica y en el marco de dicho conflicto: ¿cuál es la ganancia de los trabajadores? ¿Los trabajadores no tienen derecho a participar de las ganancias que se generan en este nuevo sistema porque no contribuirían —en la lógica tecnocrática- a generarlas?

Para nosotros esta es otra falacia en línea con paradigmas deslaboralizadores.

La riqueza la constituyen el capital y el trabajo. La tecnología no es solo producto del capital. Hubo y hay seres humanos que contribuyeron y contribuyen al desarrollo tecnológico. Sin embargo, la tecnología puede ser herramienta de un fundamento discursivo para un debilitamiento de lo humano, al decir mayoritariamente, de los y las trabajadoras. Este es un debate en sí mismo que implica la posibilidad de alinearse en distintas lógicas. Y, prima facie, se lo esgrime en un sentido desprotectorio. 
Todo lo expuesto nos corrobora que el debate no es sólo sobre el futuro del trabajo. Entendemos que, en tono de pensamiento crítico, debería ser, social, económico y sobre todo, en lo que nos ocupa, jusfilosófico. Simplificando: en una lógica ortodoxa económica, propia de la hegemonía neoliberal, solo el capital generaría riqueza. Por nuestra parte, sostenemos que se debería reflexionar con más profundidad acerca de qué ocurre en torno a los datos y su manejo. Sobre todo, cuando hablamos de datos personales; en una lógica de recolección y procesamiento de datos. Porque, cabe recordar, se recogen fundamentalmente datos personales.

En las clases, destaco a los estudiantes que casi inmediatamente luego de pasar por un lugar para ir a dar clase a una universidad que está fuera de la ciudad en que habito, recibo en mi celular publicidades de los lugares por donde atravesé; y es sabido que, si se ingresa a determinadas páginas web, los algoritmos le harán llegar propuestas o productos vinculados a esos sitios. No sería un problema que se administren datos - al menos algunos-, es una realidad parte del presente; pero sí podemos empezar a pensar en cómo regularla para evitar abusos.

Esta es otra idea central en cualquier lógica protectoria: la regulación: palabra maldita en los paradigmas neoliberales.

De hecho, el Manifiesto de Barlow (1996) sobre la Declaración de independencia del ciberespacio, es un muy claro ejemplo para explicar esta lógica.

Decimos entonces que la big data no es solo tecnología. Esto quizás suene evidente, pero cotidianamente no escuchamos este debate. La big data parece que solo fuera tecnología. La digitalización prioritariamente se dimensiona como tecnología. Pero algunos hablamos de un problema filosófico más que técnico.

Cuando se puso en funcionamiento la máquina de vapor hace alrededor de doscientos años, no fue solo tecnología. Fue tecnología en cuanto cambió la forma de producir energía, pero cambió la forma de organizar del trabajo y de producir en general. Sin esa máquina de vapor y otras máquinas como la de hilar, o la cosechadora no existiría tampoco —amén de otros factores-, probablemente, lo sindical.

La revolución industrial dió origen a los sindicatos, primigeniamente, en Inglaterra tal como los conocemos en sus aspectos fundamentales hoy en día. Modificó las formas de organización de la producción, y sin proponérselo, aglutinó a los trabajadores que, fustigados por el nuevo sistema, se organizaron para la defensa de sus intereses. El fenómeno industrial fue y es determinante para el surgimiento de las organizaciones sindicales.

La big data, aunque con especificidades diferenciadoras, es un fenómeno industrial. Simultáneamente, es un fenómeno social y económico, pero sobre todo cultural en términos filosóficos. Debe estar entonces, instrumentalmente ser vinculado con la organización y a la representación de los trabajadores.

En síntesis, los sindicatos deben reformular estrategias de representación y defensa de los intereses laborales, en especial, y sociales, en general, ante la big data.

\section{LA BIG DATA Y LA REGULACIÓN}

La big data atiende la conducta humana. Cuando se profundiza su estudio, se puede apreciar lo expuesto.

Se habla, al decir de Eric Sadin (2018: 144), de una "industria de la vida". Sintetizando dicho concepto, se trataría a partir de los datos de atender y dar respuesta hasta a las mas pequeñas conductas cotidianas. Y esto no se haría solo por una cuestión meramente de utilidad o servicio, sino por un interés económico-Ello, a su vez, implicaría la valoración y atención o desatención de determinados aspectos en función de la obtención o no de ganancia.

En esta instancia, nos permitimos recordar rápida y brevemente el concepto de biopolítica de Foucault (2007). Ese concepto, sintetizándolo muy apretadamente, alude al control social sobre 
los cuerpos y las vidas. El desarrollo tecnológico puede brindar muchos beneficios, pero también es una herramienta increíble de control, con evidentes potencialidades de autoritarismo.

Retornando al rol del Estado, no solo éste puede ser autoritario. Quizá estemos acercándonos a la posibilidad y el escenario en que el autoritarismo no provenga de él o no solo de él.

Al respecto, al referirnos a su rol en el siglo XX y en el siglo XXI, resulta pertinente recordar y precisar el concepto de ciudadanía que acuñara Marshall (1998: 13 y ss.). Es un concepto que tiene una integralidad a considerar.

Marshall sostenía que el siglo XVIII fue el siglo de los derechos civiles, el XIX de los derechos políticos y el XX el de los sociales. Poniendo al siglo XXI entre signos de pregunta, nos cuestionamos. ¿Vamos a construir una ciudadanía plena con los tres componentes o una ciudadanía sesgada? ¿Qué institucionalidad y hegemonía haremos o permitiremos que se configure, reconfigure o conserve? ¿Qué cambios y regulaciones debemos debatir?

En adelante, cuando se hable de ciudadanía, seguramente referirá a una ciudadanía digital. Y colegimos que la misma estará vinculada al acceso a las tecnologías. Pero entendemos también que debería estar también vinculada a cómo se accede a ellas y para qué sirven a los seres humanos; si sirven a los pueblos, o prioritariamente a las empresas (en tanto acceden, analizan y procesas los datos de las personas), o a ambos.

Estas son reflexiones que tenemos que poner sobre la mesa y especialmente en las mesas de negociaciones. Inclusive en las negociaciones colectivas en vista a su regulación en los convenios colectivos de trabajo.

Se suele hablar del concepto de regulación versus el de desregulación. Por nuestra parte, cuando hablamos de regulación lo hacemos inclusive al referirnos a la situación en que no hay regulación formal. A menudo se presenta como tediosa a la regulación. Paradójicamente, hasta las desregulaciones se hacen a través de leyes que establecen esas nuevas regulaciones. Pero los que buscan la desregulación, por ejemplo, para no pagar impuestos, o para que no se controle como se manejan o administran los datos personales; impulsan lo que podríamos denominar como "regulaciones-desregulatorias".

También hay que tener en cuenta cómo interviene el Estado. No tememos a la palabra intervención estatal. Conceptualmente, hemos señalado (Gambacorta, 2018:61 y ss.) la diferencia entre intervención e injerencia al referirse a cuestiones tutelares de derechos laborales. Creemos que intervención e injerencia no son lo mismo, por sus diversos aspectos, características y proyecciones.

Por ejemplo, en un artículo de The Economist de marzo de 2018, cuando se referencia la guerra comercial entre China y a EEUU, se destaca que China es el país que esta teniendo un mayor control frente a los procesos de digitalización. Esto, porque es el propio Estado chino quien está marcando determinadas reglas y fijando pautas, límites y regulaciones a las grandes empresas vinculadas a la big data. Frente a esto se encontraría el modelo de "mercado puro" adoptando por EEUU, que lo ha hecho apartarse de la lógica de inversiones estatales, y de coordinación entre las inversiones publicas y privadas para el desarrollo de tecnología. Coordinación tecnológica que, por ejemplo, lo llevo a luna.

Se trata de esquemas estratégicos. Y el corrimiento de EEUU hacia la prevalencia del mercado hace que en el año 2025 quien va a estar al frente del desarrollo en materia de inteligencia artificial sea, paradójicamente, y más allá de los paradigmas neoliberales, China. El mayor desarrollo en inteligencia artificial va a quedar en cabeza de China para dicho año.

\section{COMERCIO ELECTRÓNICO, FUTURO DEL TRABAJO Y G20}

El comercio electrónico es una operatoria caracterizable como ejemplo de un actuar en el que alguien intermedia, y pareciera, que obtiene las ganancias sólo valiéndose del soporte digital. 
En línea con lo anterior, las aplicaciones permiten un intercambio de bienes y servicios que se orientaría, entre otras cosas, a desconocer la relación laboral.

Se presenta así una desvinculación primigenia de las regulaciones, y en lo que especialmente a nosotros nos interesa, de las regulaciones laborales para la tutela de los derechos de los trabajadores y trabajadoras; que vale recordarlo, también contribuyen a que tales actividades obtengan ganancias.

Cabe indicar, asimismo, que hay datos que no afectan nuestras vidas, pero hay datos que si pueden afectarlas claramente, a saber: puede haber invasiones a la intimidad, venta y compra de nuestra información para que se sigan procesando datos y obteniendo ganancias. Por eso, hemos planteado que en el marco de la big data tenemos que empezar a discutir también los derechos digitales como derechos humanos fundamentales para el presente.

En este escenario, Latinoamérica se sitúa en un contexto de vulnerabilidad. Sobre todo, porque a menudo se cae en la generalización de interpretar la realidad como si las situaciones fueran similares, en los países desarrollados que, en los que se hallan en vías de desarrollo.

Por su parte, en la agenda del G20 reunido en Buenos Aires a fines de 2018 se incluyó el futuro del trabajo o el trabajo del futuro. Una primera advertencia: nosotros no hablamos del "fin del trabajo", a pesar de las proyecciones de J. Rifkin (1997) y las actuales asociaciones con las empresas de big data. Al respecto, entendemos que no es un tema cerrado.

A nuestro juicio, merecería un análisis y reflexiones más profundas que sentencias inmediatas y deterministas. Habría que preguntarse si el "fin del trabajo" es un hecho inexorable o es una intención.

Se señala a la inteligencia artificial. No debemos desconocer que la posibilidad de neutralizar muchas cuestiones laborales con ella sería mas factible. Ahora bien, también depende del hecho que esto lo resuelva solo una parte implicada en este conflicto o si lo resolvemos y lo debatimos todos, y en particular los actores sociales involucrados a través de sus organizaciones, por ejemplo, y como específicamente debería ser, a través de la negociación colectiva. Por ello, entendemos que si hablamos del futuro del trabajo deberíamos hablar también del futuro del capital. Temática que no suele referenciarse ni relacionarse habitualmente.

No debemos olvidar que las relaciones laborales y productivas las integran prioritariamente el trabajo y el capital. Y aquí también entra el tema de la educación. ¿Qué educación? ¿En qué educamos? ¿Para qué educamos? ¿Solo los trabajadores tienen un problema —de formación- para adecuarse a las tecnologías? ¿O merecerían discutirse otras cuestiones con un enfoque no solo economicista sino también social y humanista?

En línea con lo que señalaba Umberto Eco (2016: 455 y ss.), quizás tendríamos conceptualmente que enseñar a los trabajadores, sobre todo, a entender por sí mismos la realidad. Para que así, vean ellos mismos cómo pueden adaptarse y como pueden transformarla, más que correr tras ella en función de las, más o menos relevantes, innovaciones tecnológicas que se plasman. Las cuales, tampoco puede desconocerse, que a veces son configuradas meramente con la intencionalidad de incrementar el consumo.

\section{CONCEPTOS Y DISCURSOS: UN POCO MÁS EN TORNO AL G20}

Señalemos algunos conceptos en torno a esta temática, para analizarlos en el necesario debate y apreciar como se construye la argumentación teórica cuando se avanza en algunas cuestiones. Por ejemplo, refiriéndose al sistema financiero se suele indicar desde el G20 (2018) que "... debe permanecer abierto, resciliente y apoyando el crecimiento".

El concepto de resciliencia expresa la capacidad de adaptarse a determinados escenarios, inclusive los adversos y salir fortalecido. Deviene interesante apreciar este concepto para analizar la construcción de la argumentación teórica y la estructuración de la discusión de determinadas cuestiones. 
Entendemos que es una forma muy interesante de decir que se van a sobrellevar las realidades locales desde lo financiero pero tratando de tener las menores regulaciones posibles, a partir de la propia adaptabilidad de dicho sistema.

Ahora bien, cuando se aprecian los conceptos en la argumentación hegemónica, suelen devenir destacables por sus implicancias.

Sostenemos que no se debería caer en el prejuicio de invalidar cualquier documento por el mero hecho de su procedencia. Al contrario, consideramos que hay que trabajar sobre esos documentos para tener en claro que cuando declaman algo (aun meros resquicios) en beneficio de las mayorías - las cuales obviamente integran los trabajadores y trabajadoras-, debería exigirse y señalarse expresamente cuando no se cumple.

Si no estudiamos tales documentos - y nos referimos a documentos de la Organización para la Cooperación y el Desarrollo Económicos (OCDE), la Organización Mundial del Comercio (OMC), el Foro de Davos, el Banco Mundial, el Fondo Monetario Internacional (FMI) pero también y desde ya la Organización Internacional del Trabajo (OIT) — probablemente no podremos refutar sus restricciones ni reclamar siquiera las señales o salvaguardas que suelen (tal vez deben) dejar en sus contenidos. Especialmente, en cuanto tendieran a favorecer, o al menos reconocer, declamativamente, la defensa de los intereses de las y los trabajadores.

Ahora bien, hay que ser cuidadosos en cómo se los estudia y en contemplar el carácter de tales documentos. Volvemos aquí a cuestionar el prevalente enfoque economicista. Es dable recordar que, si bien en el G20 hay diversos grupos de trabajo (reuniones en las que, por ejemplo, pueden participar los ministros de trabajo de los distintos países miembros) los destinatarios de sus documentos son los ministros de Economía y los gobernadores o presidentes de los bancos centrales. Es decir, la problemática del trabajo se estaría debatiendo secundariamente puesto que las problemáticas se abordan prioritariamente desde una lectura economicista-financiera -esto, sin perjuicio que se de participación y presencia a la OIT o de la existencia del propio grupo laboral L20 (Labour 20).

\section{CONCLUYENDO: DESAFÍOS ANTE UN NUEVO MODELO DE ACUMULACIÓN}

Para finalizar, creemos que tenemos la necesidad de dar, sobre todas las cosas, un enfoque sistémico a la denominada cuarta revolución industrial. Esta cuarta revolución industrial que, genera angustia y dificultades por su velocidad, que siempre es mayor que nuestra propia capacidad de comprensión inmediata. Sin embargo, eso no significa que cuando comencemos a entenderla mejor, no nos podamos plantear cómo regularla y encausarla también a favor de los trabajadores.

En síntesis, estamos ante un nuevo modelo de capitalismo, pero ello no implica que los otros hayan desaparecido. Hay un nuevo modelo de capitalismo, un nuevo modelo de acumulación.

Como todo modelo de acumulación necesita apropiarse originariamente de una serie de ideas y significantes para poder consolidar su hegemonía; y a partir de su acumulación y forma de organización, terminar volcándola (no tiene muchos problemas para hacerlo porque ya es prevalente y cuasi monopólica) en un modelo de implementación. Es un nuevo modelo de acumulación en un escenario que algunos llaman anarcocapitalismo (lo cual tampoco es nuevo y guarda relaciones con la escuela austríaca de economía).

La disputa es en el Estado, por el Estado y sobre el Estado. Y tal vez más allá del Estado. Si el neoliberalismo puede controlar el Estado y la institucionalidad estatal, hará todo lo posible para desmantelar el Estado, sustituyéndolo; y tal vez, hasta privatizando una nueva institucionalidad.

Hoy están mejor dadas las condiciones para minimizar la existencia, y de ser posible, en esta lógica, hacer desaparecer el Estado. 
Creemos que ante este novedoso y complejo escenario las organizaciones sindicales tienen un trabajo propio que cumplir. Manteniendo su autonomía, tienen que impulsar una visión estratégico-política; la cual, si bien no es necesariamente partidaria, sí es contundentemente política.

Cuando vemos el accionar de las empresas, ellas tienen un accionar político sobre legisladores, ministros, magistrados; en síntesis, sobre el poder del Estado. Las organizaciones sindicales, en general, y en especial cuando no pueden llevar adelante solas sus reivindicaciones, tendrían que articular alianzas políticas, para que, si éstas inciden o llegan al control del Estado, reconozcan, consoliden y amplíen sus derechos.

Lo antes expuesto no significa que la lucha sindical no vale por si sola, pero la lucha sindical requiere; simultáneamente, una construcción política y una estrategia política sobre el Estado para la definición de un modelo determinado de sociedad.

Se trata de plasmar regulaciones tutelares, operativizándolas desde el Estado pero sin dejar de respetar las correspondientes autonomías. Con un enfoque crítico tutelar de las relaciones laborales ante una hegemonía deslaboralizadora que, se manifiesta en formas diversas, particularmente, con las nuevas tecnologías. Reiteramos que, a la vez, es una cuestión estratégica de, desde y para las organizaciones sindicales.

Si no avanzamos en una mayor regulación de la administración y uso del mercado de datos, y en una reconfiguración de la centralidad del trabajo, así como de los consecuentes derechos humanos digitales difícil nos va a ser integrarnos inclusivamente al nuevo escenario que se resume en la big data.

\section{BIBLIOGRAFÍA}

AGENCIA LATINOAMERICANA DE INFORMACIÓN (2017). Internet ciudadana o monopolios. América Latina en movimiento, núm. 528/529, pp. 1-50. Quito: ALAI.

BARLOW, J. (1996) Una declaración de la independencia del ciberespacio. Davos. Broockman, D.; Ferenstein, G. y Malhotra, N. (2017) “Wealthy Elites' Policy Preferences and Economic Inequality. The Case of Technology Entrepreneur" (Working Paper) This study was approved by the Stanford University Institutional Review Board, pp. 1-31.

Eco, U. (2016). De la estupidez a la locura. Buenos Aires: Lumen.

FOUCAULT, Michel (2007). Nacimiento de la Biopolítica. Curso en el Collège de France: 19781979. Buenos Aires: Fondo de Cultura Económica.

G20 (2018, julio 21-22). Comunicado reunión de ministros de finanzas y presidentes de bancos centrales del G20. Buenos Aires: G20.

GARCIA LINERA, A. (2012, febrero 19). Recuperado de http:/sinpermiso.info/printpdf/textos/la-institucionalidad-del poder-se traslad-al-mbito-plebeyo. Entrevista.

GAMBACORTA, M. (2018). La estructura de la negociación colectiva como proyección del modelo sindical vigente. Buenos Aires: UMSA.

GIRARDI, E. (2018). “Geopolítica de la inteligencia artificial. Capitalismo de vigilancia, democracias algorítmicas y un horizonte de Estados tecno-colonizados". Cuadernos del CEL, Vol. III, núm. 5, pp. 8-16. 
GROVER DORADO (h.), J. (2018, mayo). "Nuevos modelos de negocios en internet: regulación jurídica de servicios prestados a través de aplicaciones móviles. El caso "Uber". www.saij.gob.ar. Id SAIJ: DACF180094.

LAPEYRE, A. (2008). "La filosofía deleuziana de la diferencia y la escuela austríaca de economía”. Revista de Instituciones, Ideas y Mercados, núm. 48, pp. 219-242.

MARSHALL, T. y Bottomore, T. (1998). “Ciudadanía y clase social”. Madrid: Alianza.

MONBIOT, G. (2016, Abril 15). "Neoliberalism: the ideology at the root of all aur problems". Extraída desde https://www.theguardian.com/books/2016/apr/15/neoliberalism-ideologyproblem-george-monbiot

PERONARD, M. (2007). "Lectura en papel y en pantalla de computador.” Revista Signos, v.40, núm. 63, pp. 179-195.

RIFKIN, J. (1997). El fin del trabajo. Nuevas tecnologías contra puestos de trabajo: el nacimiento de una nueva era. Buenos Aires: Paidos.

SADIN, E. (2018). La silicolonización del mundo. La irresistible expansión del liberalismo digital. Buenos Aires: Caja Negra.

SENNETT, R. (2018, agosto 09). "O gratuito significa sempre uma forma de dominação". Entrevista por Anaxtu Zabalbeascoa. Extraída desde https://brasil.elpais.com/brasil/2018/08/09/cultura/1533824675_957329.html

THE ECONOMIST (2018, marzo 15). La batalla por la supremacía digital. La hegemonía tecnológica de Estados Unidos está amenazada por China.

Zuboff, S. (2015). "Big other: surveillance capitalism and the prospects of an information civilization”. Journal of Information Technology, núm. 30, pp. 75-89. 